\title{
Time-Resolved Velocities and Turbulence in the Oscillating Flow of a Pulse Combustor Tail Pipe*
}

\author{
JOHN E. DEC* \\ University of Michigan, Ann Arbor, MI \\ JAY O. KELLER \\ Combustion Research Facility, Sandia National Laboratories, Livermore, CA
}

ICHIRO HONGO

Toshiba Corporation, Yokohama, Japan

\begin{abstract}
The cyclic behavior of the oscillating velocity field in the tail pipe of a pulse combustor was studied using laser doppler velocimetry. In this flow, the oscillations result from an acoustic resonance and have amplitudes of up to 5 times the mean velocity. Oscillation frequencies were varied from 67 to $101 \mathrm{~Hz}$. Streamwise velocity and turbulence-intensity boundary layer profiles were measured to within $130 \mu \mathrm{m}$ of the wall, and transverse turbulence measurements were made to within $2 \mathrm{~mm}$. The phase relationships of the velocity, turbulence intensity, and combustion chamber pressure oscillations are compared. Velocity oscillations near the wall are found to phase lead those in the center of the pipe, creating periodic flow reversals through the boundary layer. A comparison is made between this turbulent oscillating boundary layer and the laminar oscillating boundary layer for flow over a flat plate. The effects of axial position, pulsation frequency, pulsation amplitude, and mean flow rate on the velocity and turbulence profiles are discussed. Time-resolved wall shear stresses (directly calculated from the velocity measurements) are presented and compared with those of steady turbulent flow. Time-averaged velocity and turbulence profiles are also compared with those of conventional steady turbulent flows. The time-averaged velocity profile is found to be flatter than that of steady flow at the same mean Reynolds number, and both the streamwise and transverse turbulence intensities are found to be significantly higher than those of steady flow.
\end{abstract}

\section{NOMENCLATURE}

D hydraulic diameter

$P \quad$ pressure

$P_{\text {rms }} \quad$ combustion chamber pressure, root mean square about the mean

*Currently at Sandia National Laboratories, Livermore, California
$R \quad$ hydraulic radius of the tail pipe, half the hydraulic diameter

$\overline{\operatorname{Re}} \quad$ time-averaged Reynolds number of the exhaust gas, based on $\bar{U}$ and $D$ time period of a complete cycle normalized time, $t^{\prime}=t / t_{\text {cycle }}$ streamwise velocity, instantaneous value, $U(t)=\bar{U}+U_{\mathrm{osc}}(t)+u^{\prime}(t)$

Copyright $(1991$ by The Combustion Institute

Published by Elsevier Science Publishing Co., Inc.

655 Avenue of the Americas, New York, NY 10010

$0010-2180 / 91 / \$ 3.50$ 
$u^{\prime} \quad$ streamwise turbulence intensity, instantaneous value

$\bar{U} \quad$ streamwise velocity time-averaged mean

$\langle U\rangle \quad$ streamwise velocity, ensembleaveraged value, $\langle U\rangle(t)=\bar{U}+U_{\text {osc }}(t)$

$\left\langle U^{\prime}\right\rangle \quad$ streamwise turbulence intensity, ensemble-averaged value, $\left\langle U^{\prime}\right\rangle(t)$ $=\sqrt{\left\langle\left(u^{\prime}\right)^{2}\right\rangle}(t)$

$\overline{U^{\prime}} \quad$ streamwise turbulence intensity, time-averaged mean of ensembleaveraged values

$U_{\text {osc }} \quad$ streamwise velocity, ensembleaveraged oscillating component

$U_{\text {osc-max }}$ streamwise velocity, ensembleaveraged oscillation amplitude, the maximum value of $U_{\text {osc }}$

$V$ transverse velocity, instantaneous value

$\bar{V} \quad$ transverse velocity, time-averaged mean

$\langle V\rangle$ transverse velocity, ensembleaveraged value

$\left\langle V^{\prime}\right\rangle \quad$ transverse turbulence intensity, ensemble-averaged value

$\overline{V^{\prime}} \quad$ transverse turbulence intensity, timeaveraged mean of ensemble-averaged values

$V_{\text {osc }}$ transverse velocity, ensembleaveraged oscillating component

$X \quad$ spatial variable along the axis of the tail pipe, $X=0$ is the contractionsection tail-pipe interface.

$Y$ vertical spatial variable normal to the tail pipe axis, $Y=0$ is at the wall

$Z \quad$ horizontal spatial variable normal to the tail pipe axis, $Z=0$ is the tail pipe centerline

\section{Greek Symbols}

$\delta_{\text {a.b.l. }} \quad$ acoustic boundary layer thickness for laminar flow

$\delta_{\nu} \quad$ characteristic dimension of the acoustic boundary layer for laminar flows

$\omega \quad$ oscillation frequency $(\mathrm{rad} / \mathrm{s})$

$\nu \quad$ kinematic viscosity

\section{INTRODUCTION}

Pulse combustion heating devices have many advantages over conventional systems, such as high thermal efficiencies, low pollutant emissions, and self-aspiration. Another major advantage is that convective heat transfer rates in the tail pipe are enhanced by as much as 2.5 times over those in steady turbulent flows at the same mean Reynolds number [1-3]. Mass transfer rates have also been found to be high when pulse combustors are used for drying applications [4]. These transport enhancements are believed to be caused by the strong flow oscillations, produced in the tail pipe by the acoustic resonance of the combustor. Most past research on transport processes in pulse combustor tail pipes has focused on direct measurements of the quantity being transported. These studies [1-3] have established the degree of heat transfer enhancement and the effect of flow parameters such as oscillation amplitude and frequency, but have done little to elucidate the transport mechanisms, which requires knowledge of the velocity field. In a recent work [5], the authors studied the behavior of the temperature field and thermal boundary layer in a pulse combustor tail pipe; however, to date, the fluid mechanics and behavior of the velocity boundary layer remain largely unstudied. A more complete discussion of the advantages, applications, and research needs in pulse combustion may be found in recent review articles $[4,6,7]$.

A review of the literature reveals that there are no detailed studies of the fluid mechanics in pulse. combustor tail pipes, and that most studies of other oscillating turbulent flows have been performed on flows in which the amplitude of the velocity oscillations is less than the mean velocity (i.e. nonreversing flows). These studies include air flows [8-10] with frequencies up to $62 \mathrm{~Hz}$ and maximum oscillation amplitudes of $34 \%$ of the mean velocity, and liquid flows [11-13] with frequencies up to $2 \mathrm{~Hz}$ and a maximum oscillation amplitude (outside the boundary layer) of $25 \%$ of the mean. It is doubtful that these studies are applicable to flows such as those in a pulse combustor tail pipe, where the amplitude of the velocity oscillations is typically $4-5$ times the 
mean velocity causing periodic flow reversals. Heat transfer studies $[1,14]$ indicate that the transport mechanisms change when large flow reversals occur; the rate of convective heat transfer was found to decrease or remain constant when the oscillation amplitude was less than or about the same as the mean velocity, while heat transfer rates increased when the oscillations were larger than the mean velocity. A study of an air flow with periodic reversals was performed by Bogdanoff [15], but only cycle-averaged profiles and no turbulence measurements are presented. In an earlier work [16], the authors reported time-resolved measurements of the bulk flow in a pulse combustor tail pipe. However, measurements were made for only a limited number of operating conditions, at one axial location, and no boundary layer measurements were made. Various approaches to modeling oscillating turbulent flows, and the comparison of these models with experimental data are reviewed by Telionis [17]. Most models and all experimental data presented by Telionis are for flows with nonreversing oscillations, although some attempts have been made to model flows with at least partial reversal. He notes that more information on the statistics of the turbulent boundary layers should be provided by experimentalists to aid in the development of new models.

The objective of the current study is to investigate bulk flow, turbulence, and boundary layer behavior of the periodically reversing flow in a pulse combustor tail pipe. Velocity oscillation amplitudes were varied from 0 (steady flow) to more than 5 times the mean velocity, with frequencies ranging from 67 to $101 \mathrm{~Hz}$. Laser doppler velocimetry (LDV) was used to make temporally and spatially resolved gas velocity measurements throughout the tail pipe. Measurements were made to within $130 \mu \mathrm{m}$ of the wall, allowing resolution of the oscillating boundary layer and direct calculation of temporally resolved wall shear stresses. This study provides an increased understanding of the fluid mechanics in pulse combustor tail pipes and insight into the mechanisms responsible for the enhanced scalar transport. This understanding of the tail pipe flow is important in the overall design of pulse com- bustors because both the fluid mechanics and heat transfer in the tail pipe are coupled to the combustion process through the acoustic resonance of the device. In addition, this study provides a data base for predictive computer models of pulse combustors [18-21], allowing results to be verified and providing necessary input parameters such as wall shear stress. Although performed in a pulse combustor tail pipe, this study may also aid modelers of other periodically reversing turbulent flows for which little experimental data exist.

\section{PULSE COMBUSTOR BACKGROUND}

Pulse combustors are devices in which the combustion process drives an acoustic resonance. For the combustor used in this study the acoustic resonance is roughly that of a quarter-wave tube with variable area. An outline drawing of the pulse combustor and plots of the maximum oscillating pressure and velocity waves generated by the acoustic resonance are given in Fig. 1. At the combustion chamber end are a pressure antinode and a velocity node, whereas at the tail pipe exit are a pressure node (constant at atmospheric) and a velocity antinode. The acoustic resonance creates velocity oscillations superimposed on the
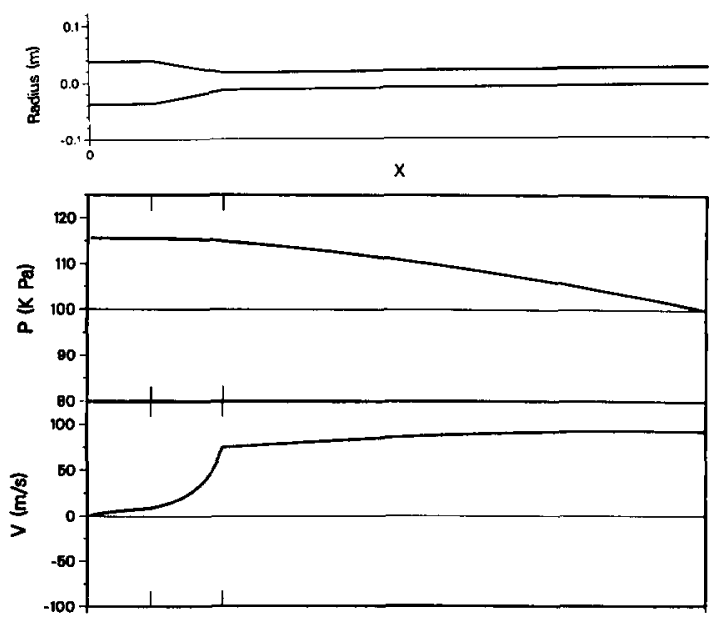

Fig. 1. Outline drawing of the pulse combustor, and typical plots of the oscillating pressure and velocity distribution at times of oscillation maximum. 


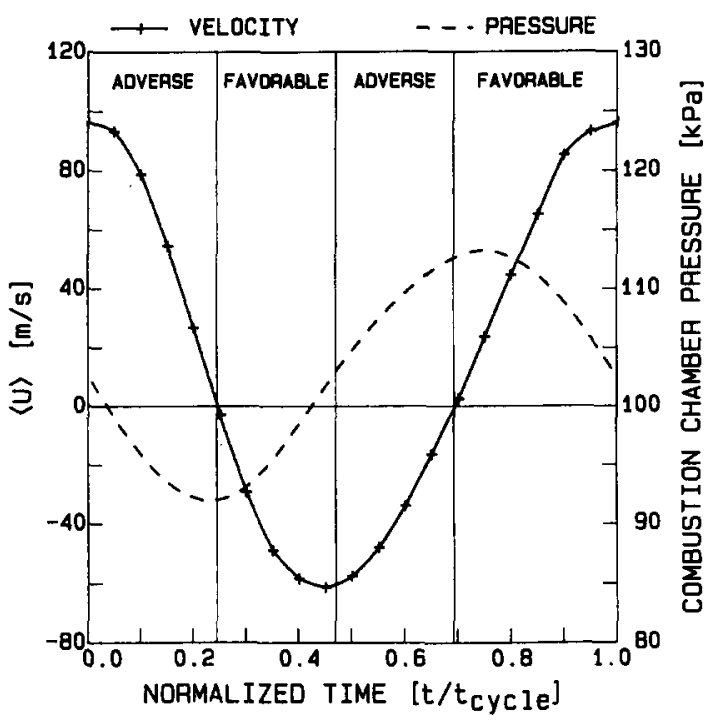

Fig. 2. Streamwise velocity and combustion chamber pressure during a combustor cycle. The velocity measurements were taken at the tail-pipe centerline, $540 \mathrm{~mm}$ from the tail pipe entrance at a combustor frequency of $83 \mathrm{~Hz}$ and $P_{\mathrm{rms}}=$ $7.4 \mathrm{kPa}$. "Adverse" and "Favorable" refer to direction of the axial pressure gradient with respect to the velocity.

mean flow of combustion products through the tail pipe. The amplitude of these velocity oscillations is greatest at the tail pipe exit and decreases sinusoidally toward the tail pipe entrance. Because of the area change between the combustion chamber and tail pipe the velocity oscillations are still significant at the tail pipe entrance (Fig. 1).

The combustion chamber pressure and tail-pipe velocity oscillations for a typical operating condition are shown in Fig. 2. These values are plotted against time normalized by the period of a complete cycle. Both waveforms are sinusoidal and are out of phase by approximately one quarter of a cycle. This is in agreement with acoustic theory for quarter-wave-tube resonance. Acoustic wave equations show that the amplitude of the velocity oscillations in the tail pipe is directly related to the amplitude of the combustion chamber pressure oscillations. This prediction has been experimentally verified in a pulse combustor by Dec and Keller [16]. In the present article the term pulsation amplitude refers to the amplitude of both the velocity and pressure oscillations.

\section{EXPERIMENTAL FACILITY AND DIAGNOSTICS}

\section{Experimental Facility}

The pulse combustor used in this study was a "Helmholtz" type with an external flapper valve. A schematic of this combustor is shown in Fig. 3. This combustor consists of an axisymmetric injection or "mixing" chamber, and square cross-section combustion chamber, contraction section and tail pipe. Acoustic termination for the tail pipe was provided by a large-volume decoupling chamber. Reactants were premixed upstream of the flapper valve and injected on-axis. Mean reactant mass flow rates were metered and controlled with sonic nozzles. The reactants consisted of pure methane and air at an equivalence ratio of 1.0 , and a variable fraction of nitrogen diluent, which was used to control the energy release rate as discussed below.

The tail pipe consisted of a coaxial square Pyrex tube arrangement (Fig. 3). In this arrangement, the inner tube, which was $30 \mathrm{~mm}$ square with a wall thickness of $4.75 \mathrm{~mm}$, formed the resonance tube for the pulse combustor and carried the combustion products. The outer tube was $51 \mathrm{~mm}$ square, which provided a $5.75-\mathrm{mm}$-wide channel between the tubes on all four sides. Cooling air flowed through this square annular space between the inner and outer tubes, entering at the decoupler end of the tail pipe and exiting at the contraction section end. This tube arrangement therefore formed a counterflowing heat exchanger. The tail pipe was constructed in a modular fashion, allowing its length to be varied in $200-\mathrm{mm}$ increments from 680 to $1280 \mathrm{~mm}$, to obtain operating frequencies from 101 to $67 \mathrm{~Hz}$, respectively. This modular construction also permitted the insertion, at $200-\mathrm{mm}$ increments, of a test section, which had walls made of optical quality quartz and Pyrex for the inner and outer tubes respectively.

The following coordinate system was used in this study. $X$ is along the axis of the tail pipe, with its origin at the contraction-section, tail-pipe interface, (Fig. 3). $Y$ is the vertical coordinate with its origin at the wall surface. $Z$ is the 


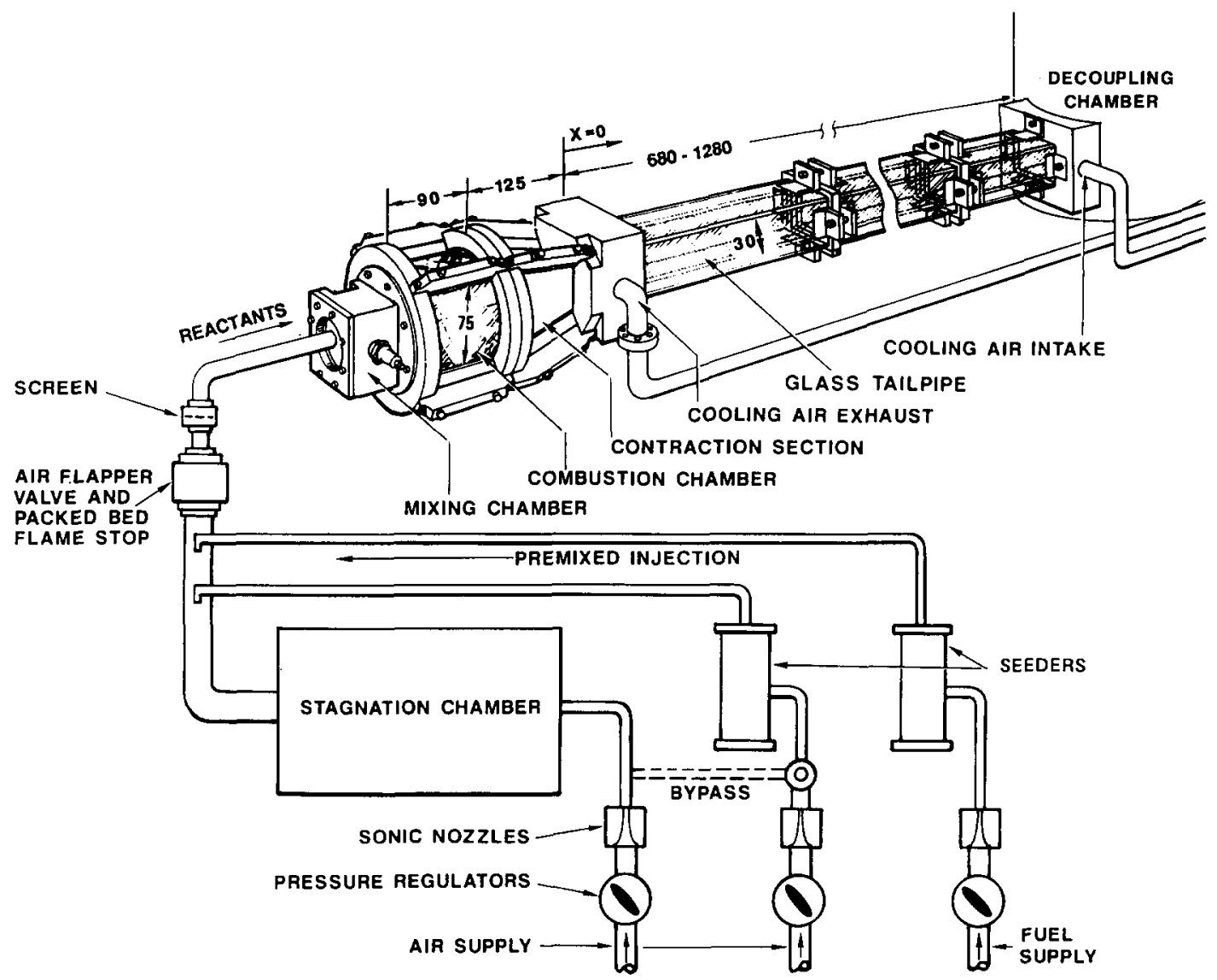

Fig. 3. Schematic of the pulse combustor facility, showing the variable-length cooled tail pipe used in this study. All dimensions are in millimeters.

horizontal coordinate with its origin at tail-pipe centerline. All profiles presented are $Y$ profiles taken at $Z=0$. Measurements at the various $X$ positions, and $Y$ profiles, were obtained by moving the entire combustor, which was mounted on a three-dimensional translation stage. All times given for cycle-resolved data have been normalized by the period of a complete cycle.

\section{Pulse Combustor Control}

A range of pulsation frequencies and pulsation amplitudes were required. Combustor frequencies were varied by changing the natural resonance frequency through variations in the tail-pipe length, and subsequent adjustment of the timing of the energy release rate. The timing of the energy release rate for this premixed system was controlled by the mixing rate of the reactants with the hot products and the chemical-reaction rate. By adjusting these two processes, the energy release was optimally timed for each natural resonance frequency, providing a maximum pulsation amplitude. Pulsation amplitudes were then decreased from this maximum by detuning the timing of the energy release through the addition of various amounts of nitrogen diluent to the reactants. Adding nitrogen slowed down the chemical reaction rate, which delayed the energy release rate. This effect could be made sufficiently strong to stop the pulsations completely and achieve steady flow. A complete discussion this methodology for controlling the pulse combustor may be found in Keller et al. [22, 23], and Bramlette [24] 
discusses jet mixing concepts in the pulse combustor. Although both strongly pulsing and steady flow conditions were obtained, the combustor would not operate in a stable manner across a wide range of intermediate pulsation amplitudes. As a result, only a limited range of pulsation amplitudes could be studied.

\section{Diagnostics}

The diagnostics used in this study were pressure transducers and LDV. Pressure transducers were used to determine the combustor frequency, and to measure the combustion chamber pressure. The root-mean-square about the mean of the combustion chamber pressure $\left(P_{\mathrm{rms}}\right)$ was used as a measure of pulsation amplitude. The combustion chamber pressure was also used to establish a synchronization trigger for the cycle-resolved LDV measurements discussed below. This trigger defined time zero of the combustor cycle and was selected to occur at the downward-going zerocrossing of the oscillating component of the combustion chamber pressure.

A two-color, four-beam LDV system was used to measure the streamwise $(U, X$ direction) and transverse $(V, Y$ direction) components of the velocity. Except for large frequency shifts, the setup was a typical forward-scatter configuration with a probe volume diameter of about $120 \mu \mathrm{m}$ and a length of $1.2 \mathrm{~mm}$. A frequency shift of 44 $\mathrm{MHz}$ was induced between the beams used to measure the $U$ component because of the large negative velocities. A large frequency shift (37 $\mathrm{MHz}$ ) was also required for the $V$ component, in order to obtain a sufficient number of doppler cycles for a signal validation, before the large $U$ velocities removed the seed particle from the probe volume. With these large frequency shifts, the resolution of the LDV counter limited the accuracy of the highest $U$ velocity $(100 \mathrm{~m} / \mathrm{s})$ to $\pm 0.32 \mathrm{~m} / \mathrm{s}$ and the accuracy of the $V$ velocities (all were near $0 \mathrm{~m} / \mathrm{s}$ ) to $\pm 0.11 \mathrm{~m} / \mathrm{s}$. A more complete discussion of the LDV system may be found in Ref. 25.

This LDV arrangement allowed measurements of the $U$ and $V$ velocity components to within $130 \mu \mathrm{m}$ and $2 \mathrm{~mm}$ of the wall, respectively. The plane of the $U$ velocity beams is parallel to the top and bottom wall of the tail pipe, permitting measurements close to the wall. The plane of the $V$ beams is normal to the wall, and in the current setup both beams are converging from the outer radius of the final focusing lens to the probe volume. With this arrangement, when the probe volume is at $Z=0$ and closer than about $2 \mathrm{~mm}$ to either the top or bottom wall, one beam is blocked, eliminating the $V$ velocity signal.

In making the $U$ velocity measurements close to the wall, thermal gradients can cause refraction of the beams and offset the probe volume location. The magnitude of this offset was estimated to be $200 \mu \mathrm{m}$ towards the wall, using an average of the time-resolved values of the thermal gradients measured in this flow by Dec and Keller [5]. This offset is not significant, because the wall location shown in the boundary layer profiles presented in this article was determined using the LDV beams. Therefore, the $Y$ positions of the data near the wall, where the thermal gradient is nearly linear, are correct. The positional error arises in the region from $Y=1.5$ to $3 \mathrm{~mm}$ where there is curvature in the thermal profile, so that for $Y>3 \mathrm{~mm}$, the location of the data is offset by $200 \mu \mathrm{m}$. In the region of $Y>1.5 \mathrm{~mm}$ the velocity gradients are small, so the positional uncertainties caused by thermal refraction were neglected.

\section{Terminology and Data Acquisition}

In an oscillating turbulent flow, the instantaneous values of both velocity components ( $U$ and $V$ ) are defined to be composed of three terms. The notation for representing these terms and the appropriate ensemble averages is presented for the streamwise velocity, with the same notation applying to $V$ :

$$
U(t)=\bar{U}+U_{\mathrm{osc}}(t)+u^{\prime}(t)
$$

where $U(t)$ is the instantaneous value, $\bar{U}$ is the long-time average mean, $U_{\text {osc }}(t)$ is the periodic or oscillating component caused by the acoustic resonance, and $u^{\prime}(t)$ is the instantaneous value of the turbulent fluctuations, which are superim- 
posed on the periodic oscillation. An ensemble average of the velocity over many cycles yields

$\langle U\rangle(t)=\bar{U}+U_{\mathrm{osc}}(t)$,

where $\langle U\rangle(t)$, the ensemble-averaged value, is still a function of time, since it varies through the cycle. Because $\left\langle u^{\prime}\right\rangle(t)=0$, the ensemble-averaged intensity of the turbulent fluctuations is given the symbol $\left\langle U^{\gamma}\right\rangle(t)$, which is defined as

$$
\left\langle U^{\prime}\right\rangle(t)=\sqrt{\left\langle\left(u^{\prime}\right)^{2}\right\rangle}(t) .
$$

In the above discussion, the functional dependence on time has been noted for clarity for all appropriate terms. Since $\langle U\rangle,\langle U\rangle$, and $U_{\text {osc }}$ are always functions of time through the cycle, the functional dependence will not be noted in the remainder of this article. The maximum value of $U_{\text {osc }}$ is the amplitude of the velocity oscillation and is denoted by $U_{\text {osc-max }}$.

The following method was used to measure the various velocity terms. The period of the cycle was divided into 20 time bins of equal length. An external clock, which was reset to a time of zero with each synchronizing trigger from the combustion chamber pressure, was connected to the laboratory computer. Each time the computer received a valid velocity realization, the elapsed time on the clock was also recorded. When the available computer memory was filled, the data were sorted into appropriate time bins based on their time of arrival, and a probability distribution function (pdf) was calculated for each time bin. The process was then repeated until each pdf contained a minimum of 400 individual velocity realizations. Since the probability of a particle arriving in the probe volume is proportional to the absolute value of the velocity, when the pdfs at cycle times near zero velocity had 400 realizations, most pdfs had more than 1000 realizations. The mean of each time-bin pdf is then a measure of $\langle U\rangle . \bar{U}$ was obtained by averaging $\langle U\rangle$ over the cycle, and $U_{\text {osc }}$ was obtained by subtracting $\bar{U}$ from $\langle U\rangle$. Similarly, standard deviation of each pdf provides a measure of $\left\langle U^{\prime}\right\rangle$. Cycle-to-cycle variation will be included in this measurement of the turbulence intensity; however it is considered negligible, since turbulence intensities near time zero, close to the synchronizing trigger, are in good agreement with those late in the cycle (Fig. 9). The time-averaged turbulence intensity $\left(\overline{U^{\prime}}\right)$ was obtained by averaging $\left\langle U^{\prime}\right\rangle$ over the cycle.

An identical data acquisition and reduction procedure was used for the transverse velocity. The mean and oscillating components of the transverse velocity ( $\bar{V}$ and $V_{\text {osc }}$ ) were on the order of the LDV resolution and were assumed to be zero.

In the pulse combustor tail pipe, the large temporal gradients of the streamwise velocity create a significant velocity variation across some time bins. Therefore, the mean and rms of the streamwise velocity pdfs must be corrected for temporal gradient broadening and temporal seed biasing, before they are accurate measures of the cycle-resolved velocity and turbulence. This was not necessary for the transverse velocity, since temporal gradients and biasing errors are small. This temporal gradient correction technique, which may be derived by adapting spatial gradient broadening corrections of Kreid [26], has also been used by Morse et al. [27] in studying the oscillating flow in reciprocating engines. A more complete discussion of this correction technique and examples of the magnitude of the correction are included in the appendix. All of the data presented in this article have been corrected for these effects. The cycle-averaged mean velocities and turbulence values were obtained by integrating the corrected means and rmss of the pdfs over the cycle.

Spatial velocity gradients through the probe volume can also create' LDV errors, commonly referred to as spatial-gradient broadening errors. At most cycle times and spatial locations spatial gradients were sufficiently small that errors were negligible. Spatial-gradient errors will be largest near the wall at cycle times when the velocity oscillation is near maximum or minimum, because at this location and time the velocity is low and velocity gradients are large. Maximum errors of $15 \%$ for $\langle U\rangle$ and $30 \%$ for $\langle U\rangle$ were estimated. Since only three or four data points per cycle at one or two $Y$ locations had errors of this magnitude, and all others were significantly less, the 
LDV measurements were not corrected for spatial gradient broadening errors.

\section{RESULTS AND DISCUSSION}

Most data to be presented were obtained at a typical combustor operating condition and axial location, which will be referred to as the base condition and base location. The base condition is a tail pipe length of $880 \mathrm{~mm}$, a frequency of 83 $\mathrm{Hz}$, a $P_{\mathrm{rms}}$ of $7.4 \mathrm{kPa}$, and a mean flow Reynolds number (based on $\bar{U}$ ) of 3750 . The base location is $X=540 \mathrm{~mm}$. Data were also taken at other operating conditions and locations and will be compared to those of the base condition and location. Velocities and spatial variables are presented in dimensional units, except as noted, because the appropriate nondimensional groups are unknown for these boundary layers, and there appears to be no accepted standard in the literature. Also, no attempt was made to reduce the frequencies to nondimensional form because the proper similarity variable in turbulent flow is unknown [17].

The fundamental streamwise velocity oscillation on the tail-pipe centerline, and its relation to the combustion chamber pressure oscillation, for the base condition and location, are shown in Fig. 2. Due to the nature of the acoustic resonance and the definition of time zero, the tail-pipe velocity is at its maximum, $95 \mathrm{~m} / \mathrm{s}$, at the start of the cycle. The velocity then decreases, reaching zero at about time 0.25 , and continues to decrease until a minimum of $-60 \mathrm{~m} / \mathrm{s}$ is reached at time 0.45 . From this minimum, the velocity increases back to the maximum at time 1.0, passing through zero at about time 0.7 . The mean velocity is 16.3 $\mathrm{m} / \mathrm{s}$. Keeping in mind that the tail-pipe exit pressure is constant, it can be seen that velocity flows against the pressure gradient (adverse) from time 0.0 to 0.25 , and from time 0.45 to time 0.7 , while the pressure gradient is favorable from time 0.25 to 0.45 and 0.7 to 1.0 . Changes in the pressure gradient from favorable to adverse are necessary for pressure driven velocity oscillations. The times and velocities noted in the above discussion were typical for all operating condi-

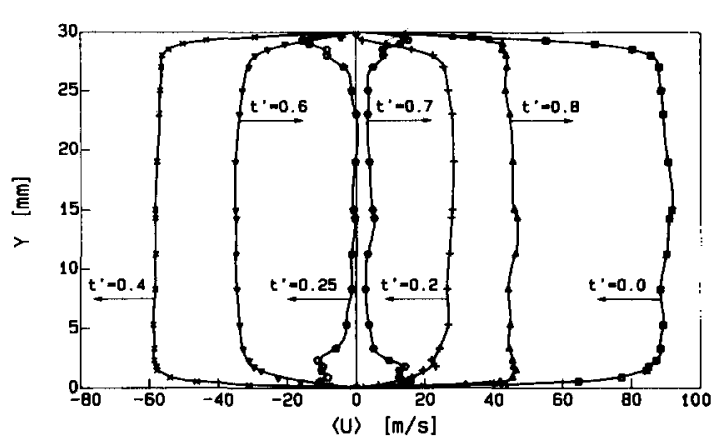

Fig. 4. Time-resolved velocity profiles across the tail pipe. $t^{\prime}$ is time normalized by the period of a cycle. The direction of the arrows indicates the direction of the temporal velocity gradient. Base operating condition and base location.

tions with similar pulsation magnitudes, although small variations occurred.

\section{Time Resolved Velocity Profiles}

Full profiles of $\langle U\rangle$ at various times during the cycle are shown in Fig. 4. All data presented in this subsection are for the base condition and location; however, the temporal behavior seen in these figures is typical of all pulsation frequencies, pulsation amplitudes, and axial locations studied. The time of each profile is noted on the figure, and the direction of the arrows indicate the sign of the slope of the velocity-time curve. Three features may be seen in these profiles. First, the profiles are flat across the center of the pipe at all times during the cycle, indicating that the centerline velocity is a good indicator of the bulk velocity behavior. Second, the flow is symmetric from top to bottom. Third, the phase of the flow near the wall leads the phase of the flow in the central region during the flow reversals (times of 0.25 and 0.7 ), as is discussed below. In view of the first two observations, the velocity profile may be adequately represented by a plot of the region near one wall, which allows the third observation to be more clearly visualized.

In Fig. 5, the $\langle U\rangle$ velocity profiles for the 6 $\mathrm{mm}$ adjacent to the wall are plotted, for all time bins recorded. The top plot shows the profiles for the decreasing velocities, times 0.0 to 0.45 , while the bottom plot shows the increasing velocities, 

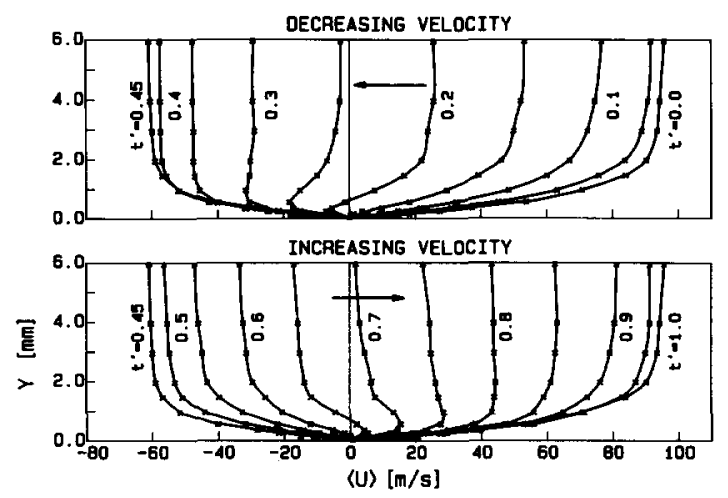

Fig. 5. Time-resolved velocity boundary-layer profiles. The number adjacent to the profiles indicate $t^{\prime}$, time normalized by the period of a cycle. Base operating condition and base location.

times 0.45 to 1.0 . As with conventional steady turbulent flows, the profiles may be divided into three regions: (1) a core region, which extends from the centerline to 3 or $4 \mathrm{~mm}$ from the wall, (2) a viscous dominated sublayer near the wall, and (3) a buffer layer between the viscous sublayer and the core. Measurements were obtained sufficiently close to the wall $(130 \mu \mathrm{m})$ to resolve the viscous sublayer. Figure 5 clearly shows the temporal development of the boundary layer during the flow reversals. During times of both decreasing and increasing velocity, it can be seen that the flow near the wall reverses earlier in the cycle than the core flow, with the result of a reversing slope of the velocity profile. This is the first known observation of this phenomenon in turbulent flow, although it is well known behavior for laminar flow (see for example, Ref. 28).

Two factors play a role in creating this boundary layer behavior in a pressure-driven oscillating flow. First, the pressure gradient is constant across the tail pipe $(\partial P / \partial Y \approx 0)$, but the fluid near the wall has less momentum than the fluid in the core. As a result, when the pressure gradient changes from favorable to adverse, the fluid near the wall, having less momentum, slows down and reverses sooner than the fluid in the core. The second reason for this boundary layer reversal is the finite penetration rate of the effect of the viscous drag at the wall (wall effect) into the fluid. This effect may be visualized by noting that as the free-stream velocity decreases from time 0.0 to 0.2 , the shape of the velocity boundary layer between 0.5 and $6.0 \mathrm{~mm}$ remains virtually unchanged, since the wall effect has only penetrated in to $0.5 \mathrm{~mm}$ at this time. Because of this, a boundary layer slope reversal must occur for the velocity to go to zero at the wall.

Although the turbulent transport affects the boundary layer behavior seen in Fig. 5, it is useful to compare the observed turbulent oscillating profiles with those of a laminar incompressible flow. The solution for the velocity field in a semi-infinite fluid, bounded by an oscillating wall, was first given by Stokes in 1851 (Stokes' second problem.) For the case of an oscillating semiinfinite fluid, bounded by a stationary wall, driven by an imposed spatially uniform oscillating pressure gradient, the solution (assuming that the flow is everywhere parallel to the wall) may be expressed as [28, 29].

$$
\begin{aligned}
\frac{U_{\text {osc }}}{U_{\text {osc }-\max }}= & \cos \left(2 \pi t^{\prime}\right) \\
& -\bar{e}^{\left(Y / \delta_{\nu}\right)} \cos \left(2 \pi t^{\prime}-Y / \delta_{\nu}\right),
\end{aligned}
$$

where $U_{\text {osc }}$ and $U_{\text {osc-max }}$ were defined previously, $t^{\prime}$ is normalized time, and $\delta_{\nu}$ is the characteristic dimension of the acoustic boundary layer, defined as

$$
\delta_{\nu}=\sqrt{\frac{2 \nu}{\omega}} .
$$

Here $\omega$ and $\nu$ are frequency in radians per second, and kinematic viscosity, respectively. The solution for the velocity in Eq. 4 is the difference between a spatially uniform oscillating velocity field (first term) and the solution of Stokes second problem (second term). Although Stokes' second problem contains no pressure field information, Eq. 4 includes the oscillating pressure gradient through the first term [29].

The depth of penetration of the wall effect on the free stream oscillations is commonly referred to as the "acoustic boundary layer," $\delta_{\text {a.b.l. }}$. Defining this boundary layer to be the distance at which the wall oscillations have decreased to $1 \%$ 


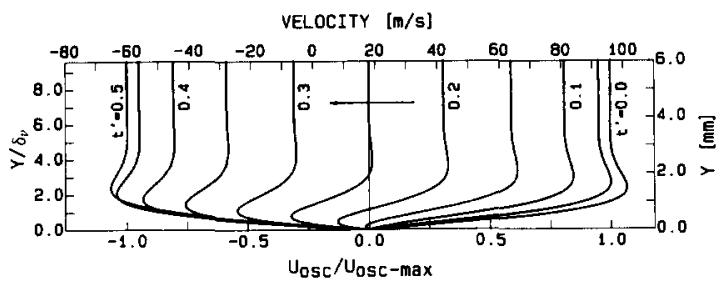

Fig. 6. Time-resolved velocity boundary-layer profiles predicted for oscillating laminar flow with no mean flow. Scales on the bottom and left are in universal dimensionless units. Scales on the top and right are dimensional scales for the base operating condition and base location, adjusted so the plot scale is the same as Fig. 5.

results in [30],

$\delta_{\mathrm{a} . \mathrm{b} .1 .} \approx 4.6 \delta_{p}$

This acoustic boundary layer thickness is equal to $2.87 \mathrm{~mm}$ for the base condition.

The results of Eq. 4 for the first half-cycle are plotted in Fig. 6. Two scales are given for each axis. At the left and bottom are the universal dimensionless variables, $Y / \delta_{\nu}$ and $U_{\text {osc }} /$ $U_{\text {osc-max }}$, while at the right and top are the dimensional variables for the base condition. These dimensional scales and the oscillation amplitude far from the wall are the same as Fig. 5, for ease of comparison. The laminar flow solution (Fig. 6), exhibits the same flow reversal through the boundary layer seen in the turbulent data (Fig. 5). It can be seen that the acoustic boundary layer thickness is a good indicator of the penetration depth of the oscillations for both the turbulent data and the laminar solution. Moreover, for the turbulent data the acoustic boundary layer thickness is comparable to the fluid dynamic boundary layer.

There are also significant differences in Figs. 5 and 6 . The laminar flow solution predicts a velocity overshoot, relative to the free stream (called Richardson's annular effect [28]), in the region from 1 to $2.5 \mathrm{~mm}$ at times of maximum and minimum oscillation amplitude, while the turbulent flow data has a smooth curve with a monotonic slope. In turbulent flow the additional momentum transport by the turbulence more rapidly dissipates the oscillations as they penetrate inward from the wall. This smoothing effect of the turbulence can be seen in the reduced sharpness of the boundary layer reversal at time 0.25 . The increased transport due to the turbulence is also evident in the slope of the profiles from 2.87 to 6 $\mathrm{mm}$. In the laminar case they are flat and vertical, while in the turbulent data a small slope remains in some profiles, even at $6 \mathrm{~mm}$.

The phase shift of the velocity oscillations from the core through the boundary layer is more clearly shown in Fig. 7, which shows $U_{\text {osc }}$ versus normalized time for several transverse locations. Moving from the core to the wall, the zero velocity crossings of the curves occur systematically earlier in the cycle. A systematic decrease in oscillation amplitude is also seen, and, although the plots for $Y$ positions between the centerline and $1.5 \mathrm{~mm}$ are not given, they too show a systematic decrease. This is in contrast to the predicted results for laminar flow in which the average oscillation amplitude is larger at about half the acoustic boundary layer thickness, $Y / \delta_{\text {v }}$ $\approx 2.0$, than in the free stream, due to the large velocity overshoots at times 0.0 and 0.5 noted in the discussion of Fig. 6. A discussion of this phenomenon may be found in Ref. 28 .

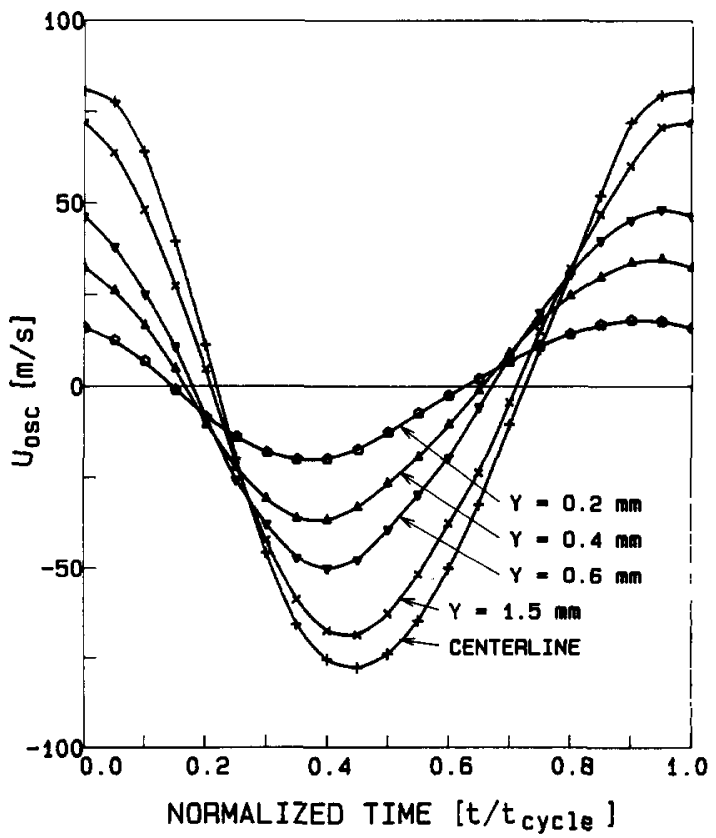

Fig. 7. Streamwise velocity oscillations at various $Y$ locations through the boundary layer. Base operating condition and base location. 


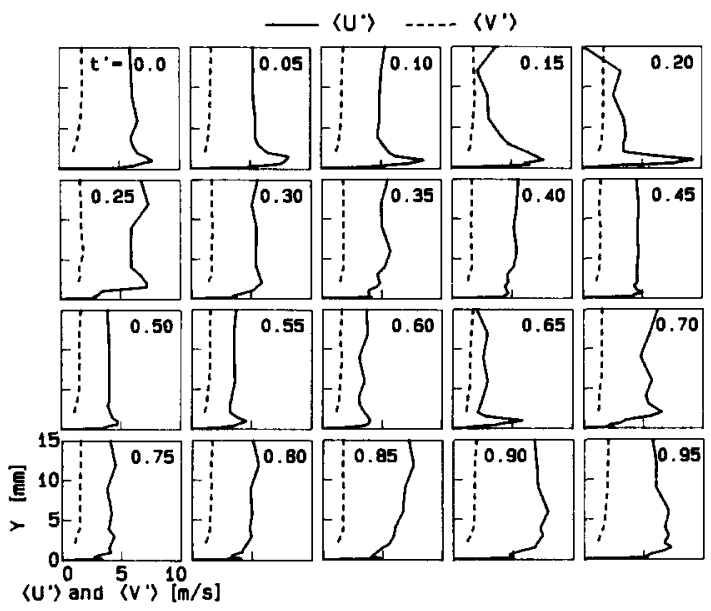

Fig. 8. Time-resolved streamwise and transverse turbulence profiles. The number in each box is $t^{\prime}$, time normalized by the period of a cycle. Base operating condition and base location.

\section{Time-Resolved Turbulence Intensities}

Ensemble-averaged turbulence-intensity profiles for the base condition and base location are presented in Fig. 8. As with the above mean velocity data, the temporal behavior described in the following discussion is typical for all frequencies, pulsation amplitudes, and axial locations. Profiles of both the streamwise and transverse turbulence intensities $\left(\left\langle U^{\gamma}\right\rangle\right.$ and $\left.\left\langle V^{\gamma}\right\rangle\right)$ are presented for each time bin; normalized times are denoted by the number given in each box. As discussed previously, transverse velocities could only be measured to within $2 \mathrm{~mm}$ of the wall. The $\left\langle V^{\prime}\right\rangle$ profiles show the transverse turbulence intensity to be nearly constant across the tail pipe, at about $1.5 \mathrm{~m} / \mathrm{s}$ at all times during the cycle. There is some indication of a decrease in the magnitude of $\langle V\rangle$ at the points closest to the wall, although these data are insufficient to determine if this downward trend continues to the wall or if there is an increase near the wall, as occurs at some times with the streamwise turbulence intensity.

Significant cyclic variation occurs in the profile shape of the streamwise turbulence intensity. At any given time during the cycle, the turbulence intensity is uniform across the core region of the flow, but the behavior in the boundary layer region has two distinctly different modes. From times 0.0 to 0.2 and 0.5 to 0.65 , when the pressure gradient is adverse (Fig. 2), the intensity increases from the core value to a maximum in the boundary layer before going to zero at the wall. In contrast, from times 0.25 to 0.4 and 0.7 to 0.95 the intensity drops from the core value throughout the boundary layer. In steady flows, adverse pressure gradients are known to thicken the boundary layer and lead to instability, while favorable pressure gradients decrease boundary layer thickness and increase stability [31].

The intensity of the streamwise turbulence also has significant cyclic variation as shown in Fig. 9, in which $\left\langle U^{\rangle}\right\rangle$is plotted against normalized time through the cycle for three transverse $(Y)$ ranges corresponding to the three regions of the velocity profile described above. For all $Y$ positions greater than $0.5 \mathrm{~mm}$, a significant local increase or "spike" in the turbulence intensity may be noted at times of zero-velocity crossings (in the core at times 0.25 and 0.7 , and in the buffer layer, where the velocity oscillations phase lead the core, at times 0.2 or 0.25 and 0.65 or

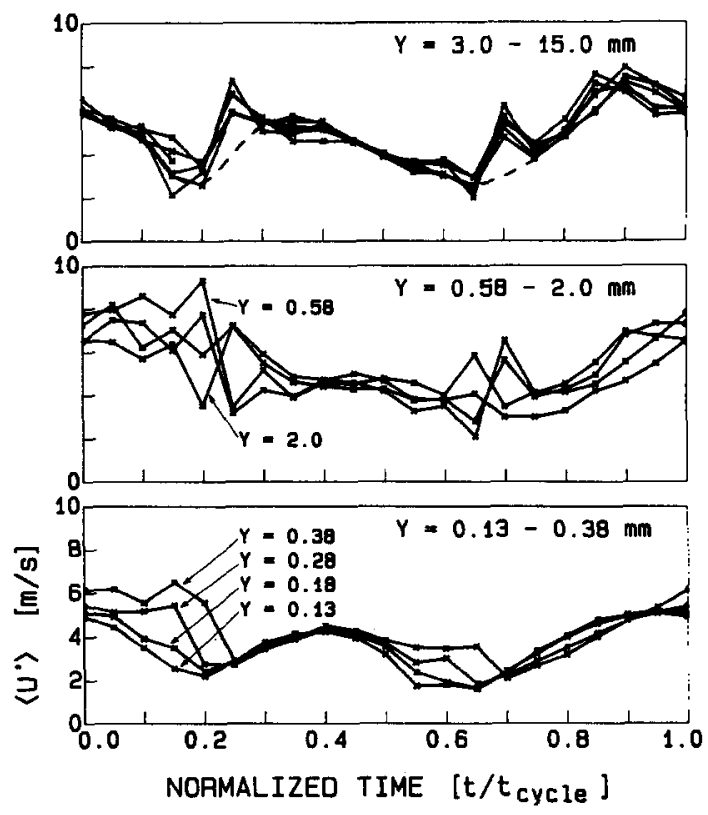

Fig. 9. Streamwise turbulence as function of cycle time for three transverse regions. The dashed line in Fig. 9a is a spline fit to the data with the points at normalized times 0.25 and 0.7 (the zero-velocity crossings of the streamwise velocity) removed. Base operating condition and base location. 
0.7). As discussed in the Appendix, the turbulence-intensity corrections for gradient broadening and biasing are very large at these times, and these spikes might only be an artifact of the diagnostic and correction technique. Alternatively, there may be short bursts in the turbulence intensity associated with the flow reversal. Similar spikes were seen at all operating conditions, and the relative intensity of the spikes is very consistent at all $Y$ locations in the core, indicating that either they are real, or there is a large systematic error in the velocity correction at these cycle times.

The dashed line in the top plot in Fig. 9 is a cubic spline fit to a typical $\left\langle U^{\prime}\right\rangle$-time history in the core with the data points at the zero-velocity crossings eliminated. By eliminating the spikes, the basic trends of the turbulence intensity in the core through the cycle may be seen. The turbulence intensity gradually decreases, as does the velocity, from time 0.0 to 0.2 . After the flow reversal at time 0.25 , the velocity increases in absolute value, and $\langle U\rangle$ increases to a local maximum about time 0.3-0.35. $\langle U\rangle$ then decreases again gradually to time $\mathbf{0 . 6 5}$, which is just prior to the second flow reversal. Again, following this flow reversal, $\langle U\rangle$ increases rapidly to a maximum about time $0.85-0.9$, after which it gradually decreases towards the first zero velocity crossing. Thus, the local maxima of the turbulence intensity phase-lead the maximum of the absolute value of the velocity by $0.10-0.15$ of a cycle, while the local minima phase-lead the times of zero velocity by only 0.05 of a cycle. The relative magnitudes of the maxima, 5.5 and 8 $\mathrm{m} / \mathrm{s}$, are proportional to the relative absolute magnitudes of the velocity minimum and maximum, -60 and $95 \mathrm{~m} / \mathrm{s}$. These turbulence-intensity oscillations occur nearly simultaneously, and with the same magnitude, across the core ( $Y=3$ to $15 \mathrm{~mm}$ ), indicating that the turbulence is being generated locally. During the combustor cycle, the flow is periodically accelerating and decelerating in the $X$ direction, uniformly across the core. The streamwise component of the turbulence will be periodically stretched and compressed by these accelerations and decelerations, and have a resulting oscillation in intensity.
In the buffer layer, the characteristic $\left\langle U^{\rangle}\right\rangle$-time behavior changes from that of the core to that of the viscous sublayer. In this viscous dominated sublayer, $\left\langle U^{\prime}\right\rangle$ has a different temporal behavior, and the spikes at the times of zero velocity crossing are no longer present. The two curves closest to the wall ( $Y=0.13$ and $0.18 \mathrm{~mm}$ ) are very smooth and symmetric, and the times of their local maxima (0.4 and 0.9) are in phase with the velocity oscillation and spatial-velocity gradient at this $Y$ location (Figs. 5 and 7$)$. These $\langle U\rangle$ oscillations are believed to result from the periodic generation of turbulence by the velocity shear present at these times at this location in the flow. Further from the wall, $Y=0.28$ and 0.38 $\mathrm{mm}$, the adverse pressure gradients are also contributing to the turbulence, and a higher turbulence intensity persists after the local maxima from times 0.0 to 0.2 and 0.45 to 0.6 .

\section{Variation With Axial Position}

Although the temporal behavior of the velocity profiles and turbulence intensities is similar at all axial locations, the mean flow rate and velocity oscillation amplitude vary and some entrance and exit effects exist. These effects are shown in Fig. 10 and 11, for the base condition. In Fig. 10a, the tail-pipe centerline velocity, which, as noted above, is a good indicator of the bulk velocity, is plotted as function of distance along the tail pipe. A steady decrease in velocity occurs as a result of heat transfer along the pipe. This heat transfer has been found to result in a 500-K temperature drop between $X=140$ and $740 \mathrm{~mm}$ for this base condition $[2,5]$. Under ideal gas assumptions this temperature drop agrees well with the 7.4-m/s velocity decrease noted here. Ensemble-averaged values of $U_{\text {osc }}$ at the tail-pipe centerline through the combustor cycle are presented in Fig. $10 \mathrm{~b}$. The oscillation amplitude increases from the tailpipe entrance to the exit due to the sinusoidal distribution of the velocity oscillations of the resonant acoustic wave (Fig. 1). The amplitude increase of $19 \%$ from 71 to $82 \mathrm{~m} / \mathrm{s}$ for $X=140$ and $740 \mathrm{~mm}$, respectively, is not large. This indicates why Helmholtz resonator models, which assume slug flow in the tail pipe, have sometimes 

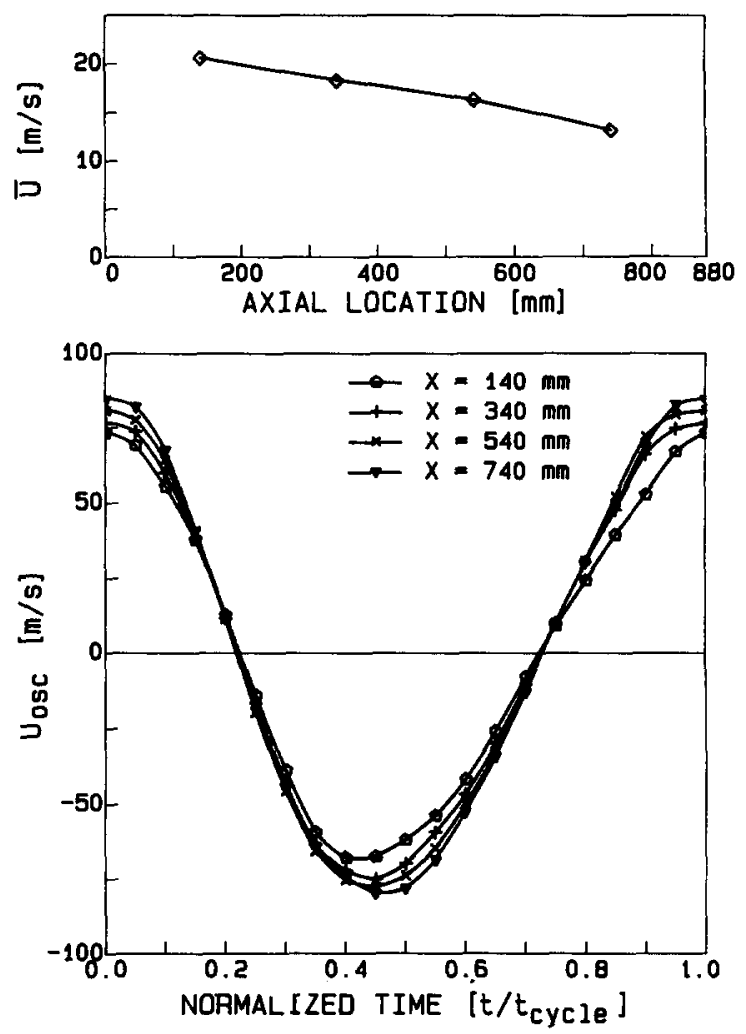

Fig. 10. Variation of the streamwise velocity at the tail-pipe centerline with axial location. Figure 10a shows the mean velocity variation due to heat transfer and Fig. $10 \mathrm{~b}$ shows the oscillating component. Base operating condition.

been found to give reasonably good predictions of pulse combustor frequencies. Figure $10 \mathrm{~b}$ also shows that near the exit of the tail pipe ( $X=740$ $\mathrm{mm}$ ) the velocity oscillations are nearly symmetric, with the minimum occurring at time 0.47 , while near the entrance ( $X=140 \mathrm{~mm}$ ) the minimum occurs at time 0.4. Measurements farther from the combustion chamber in longer tail-pipe configurations showed the minimum at time 0.5 , as would be expected for a purely acoustic resonance. This effect is believed to be due to the periodic influx of, and energy release from, the reactants in the combustion chamber, which disturb the fundamental acoustic resonance.

In Fig. 11, time-averaged streamwise velocity $(\vec{U})$ and time-averaged turbulence-intensity $\left(\overrightarrow{U^{\prime}}\right)$ profiles show the entrance and exit effects in both pulsing (base conditions) and nonpulsing flows.

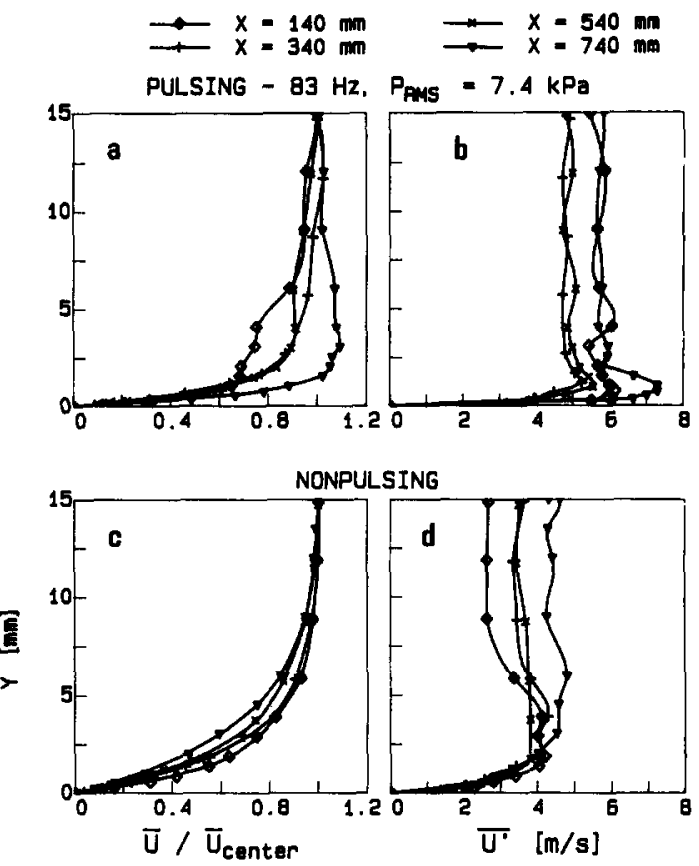

Fig. 11. Axial variation of time-averaged streamwise velocity and streamwise turbulence profiles for pulsing and nonpulsing flows. The mass flow rate is the same for the pulsing and nonpulsing data. Pulsing flow is at the base operating condition.

The $\bar{U}$ profiles are normalized by the centerline value to remove the velocity changes due to heat transfer, and to more clearly show variations in boundary layer shape. Figure 11a shows that away from the entrance and exit of the tail pipe, $X=340$ and $540 \mathrm{~mm}$, the $\bar{U}$ profile shape does not vary significantly with axial position, indicating fully developed flow. The measurement closest to the entrance, $X=140 \mathrm{~mm}$, shows a velocity defect from $Y=1$ to $6 \mathrm{~mm}$ when compared to the profiles farther from the entrance. Similarly the measurement closest to the exit, $X=$ $740 \mathrm{~mm}$, shows a relative increase in velocity for $Y=1$ to $9 \mathrm{~mm}$. These seemingly opposite effects are believed to result from the same phenomenon. During the positive-velocity half of the cycle the gases are being accelerated through the contraction section into the tail pipe entrance, where there is a sudden change in wall angle of 10 degrees. At the high periodic velocity of $90 \mathrm{~m} / \mathrm{s}$ this wall angle change is sufficient to cause a 
jetting effect, or vena contracta, which results in the gases near the centerline having a higher velocity. Similarly, at the tail-pipe exit during the negative-velocity half of the cycle, this jetting effect causes a greater negative velocity in the gases near the centerline. This greater negative velocity, when averaged over the cycle and normalized by the centerline velocity, shows up as an increased positive velocity away from the centerline. The tail pipe has a square exit into the decoupling chamber, which increases the magnitude of the effect at the exit compared to the entrance.

The pulsing, $\overline{U^{\prime}}$ profiles in Fig. $11 \mathrm{~b}$ show the same trends as the mean velocity. At $X=340$ and $540 \mathrm{~mm}$ the profiles are virtually identical, while in the entrance and exit regions the turbulence intensity is about $20 \%$ higher. This higher turbulence intensity is attributed to entrance effects, which as noted above occur at both ends of the tail pipe as a result of the reversing flow. All four profiles are flat across the core region and increase through a maximum in the boundary layer. This increase in the boundary layer is small except for $X=740 \mathrm{~mm}$, indicating a stronger effect of the jetting action and flow separation at the exit than the entrance.

In Figs. 11c and 11d, the results of nonpulsing (steady) flow in the same combustor are presented for comparison. These profiles have the same mean flow-rate as the above pulsing data, but the heat transfer rate for the steady flow is much lower [2], resulting in higher gas temperatures. As a result the axially averaged bulk velocity is higher $(21.4 \mathrm{~m} / \mathrm{s})$ but the Reynolds number is lower by $7 \%(3500)$ due to changes in viscosity. The steady-flow velocity profiles show a small, but consistent trend of boundary layer development with $X$. This is in contrast to mean velocity profiles of the pulsing data (Fig. 11a), which showed little change with $X$ in the central region of the pipe. The steady-flow $\overline{U^{\prime}}$ profiles (Fig. 11d) also show development with axial position, with an increasing turbulence intensity in the core. These steady-flow velocity and turbulenceintensity profiles shapes are not typical of classic steady turbulent flow, as is discussed later.

There are significant differences between the
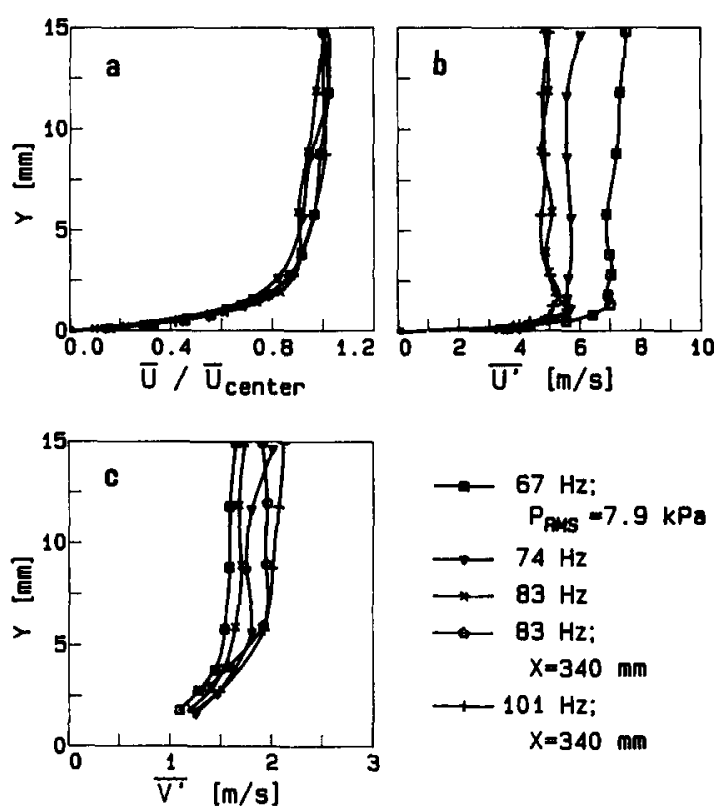

Fig. 12. Frequency variation of time-averaged streamwise velocity (a), streamwise turbulence (b), and transverse turbulence (c) profiles. $P_{\mathrm{rms}} \approx 7.4 \mathrm{kPa}$, and $X=540 \mathrm{~mm}$, except as noted.

data from the pulsing and nonpulsing flows, in both the $\bar{U}$ and the $\bar{U}^{\prime}$ profiles. In the pulsing flow the $\bar{U}$ profiles, similar to the $\langle U\rangle$ profiles, are flat across the core and drop rapidly to zero in the $3 \mathrm{~mm}$ adjacent to the wall, while in the nonpulsing flow the velocity profiles have an almost constant curvature. The differences in the $\bar{U}^{\prime}$ profiles are not as dramatic, but they also exhibit the same trends. Finally, the average turbulence intensity is lower in the nonpulsing flow than the pulsing case.

\section{Variation With Frequency}

The effects of frequency on the $\bar{U}, \overline{U^{\prime}}$, and $\overline{V^{\prime}}$ profiles are shown in Figs. 12a, 12b, and 12c, respectively. The pulsation amplitude for all these data was nearly constant, and the axial location was $X=540 \mathrm{~mm}$ for all frequencies except 101 $\mathrm{Hz}$, where it was $X=340 \mathrm{~mm}$, as noted in Fig. 12. This was necessary to avoid the exit effects, which are present at $X=540 \mathrm{~mm}$ in the $680-\mathrm{mm}$ tail pipe used to obtain the $101-\mathrm{Hz}$ data. The data in Fig. 11 show that the effect of this change of location is small. 
TABLE 1

Time-Averaged Turbulence Intensities and Wall Shear Stresses for Various Frequencies and Pulsation Amplitudes ${ }^{a}$

\begin{tabular}{|c|c|c|c|c|c|c|c|c|}
\hline $\begin{array}{c}\text { Frequency } \\
(\mathbf{H z})\end{array}$ & $\begin{array}{c}P_{\mathrm{rms}} \\
(\mathrm{kPa})\end{array}$ & $\begin{array}{c}U_{\mathrm{osc}-\max } \\
(\mathrm{m} / \mathrm{s})\end{array}$ & $\begin{array}{c}\bar{U} \\
(\mathrm{~m} / \mathrm{s})\end{array}$ & $\frac{U_{\text {osc }-\max }}{\bar{U}}$ & $\begin{array}{c}\overline{\left\langle U^{\prime}\right\rangle} \\
(\mathrm{m} / \mathrm{s})\end{array}$ & $\begin{array}{c}\overline{\left\langle V^{\prime}\right\rangle} \\
(\mathrm{m} / \mathrm{s})\end{array}$ & Wall & $\begin{array}{l}\text { Stress } \\
\text { 2) } \\
\text { Average } \\
\text { Absolute } \\
\text { Value }\end{array}$ \\
\hline 67 & 6.6 & 61.0 & 18.5 & 3.30 & 8.21 & 1.57 & -0.43 & 1.34 \\
\hline 67 & 7.2 & 65.5 & 18.8 & 3.48 & 6.77 & 1.63 &.-- &.-- \\
\hline 67 & 7.9 & 71.2 & 17.8 & 4.00 & 7.54 & 1.65 & -0.55 & 1.66 \\
\hline 74 & 7.3 & 73.4 & 17.2 & 4.27 & 6.03 & 2.01 & -0.34 & 1.50 \\
\hline 74 & 7.6 & 74.9 & 17.2 & 4.36 & 6.20 & 1.78 &.-- &.-- \\
\hline 74 & 7.9 & 76.6 & 17.1 & 4.48 & 7.25 & 1.84 &.-- &.-- \\
\hline 74 & 8.1 & 77.4 & 17.2 & 4.50 & 8.07 & 1.73 & -0.38 & 1.67 \\
\hline 83 & 7.4 & 79.3 & 16.3 & 4.87 & 4.82 & 1.73 & -0.36 & 2.00 \\
\hline 101 & 7.3 & 79.0 & 18.9 & 4.18 & 4.98 & 2.12 & -0.48 & 1.86 \\
\hline
\end{tabular}

${ }^{a}$ All velocities and turbulence intensities are at the tail-pipe centerline. $X=540 \mathrm{~mm}$, except for the $101-\mathrm{Hz}$ data, for which $X=340 \mathrm{~mm}$.

All the $\bar{U}$ profiles are similar, and, although there is some small variation in the shape with frequency, there is no consistent trend. Similarly, the $\overline{V^{\prime}}$ profiles show no consistent trend and only small variation with frequency. The higher $\overrightarrow{V^{\prime}}$ intensity at $101 \mathrm{~Hz}$ is believed to be an artifact of the $X=340 \mathrm{~mm}$ location, since the intensity for this location at $83 \mathrm{~Hz}$, also shown, is higher too. In Fig. $12 \mathrm{~b}$, a variation in the magnitude of $\overline{U^{\prime}}$ with frequency is seen, although the profile shapes are similar. For both the $83-$ and $101-\mathrm{Hz}$ cases, $\bar{U}^{\prime}$ has a value of about $5 \mathrm{~m} / \mathrm{s}$ across the core. At 74 $\mathrm{Hz}$ the intensity is slightly higher, while in the $67-\mathrm{Hz}$ case the magnitude of $\bar{U}^{\prime}$ has increased to $7 \mathrm{~m} / \mathrm{s}$. This increase is consistent across the core, and, although there is no change between the 83and $101-\mathrm{Hz}$ cases, a general trend of increasing $\overline{U^{\prime}}$ with decreasing frequency may be noted. This trend was not expected and indicates that the observed increases in heat transfer rates with frequency [2] are not a result of simply an increased turbulence intensity.

\section{Variation With Pulsation Amplitude}

The results of varying the pulsation amplitude for two frequencies, 67 and $74 \mathrm{~Hz}$, are shown in Table 1. All the velocities presented are from the tail-pipe centerline; these are good indicators of the bulk velocity, since $\langle U\rangle$ and $\left\langle U^{\prime}\right\rangle$ profiles are relatively flat. The pulsation amplitude is expressed as both $\boldsymbol{P}_{\mathrm{rms}}$ and the velocity oscillation amplitude $\left(U_{\text {osc }-\max }\right)$. These two independent measurements of the pulsation amplitude are in agreement. $\bar{U}$ is also shown, since it varied with pulsation amplitude due to thermal effects, although the mass flow rate was constant. (Temperature measurements for this flow field are presented in Ref. 5). Despite these variations, the ratio of the oscillating and mean velocities $\left(U_{\text {osc-max }} / \bar{U}\right)$ may be seen to monotonically increase with increasing pulsation amplitude. This finding is in agreement with Ref. 2 in which the heat transfer increased with pulsation amplitude and was thought to be a function of $U_{\text {osc-max }} / \bar{U}$. Also shown are average turbulence intensities, $\bar{U}^{\prime}$ and $\overline{V^{\prime}}$. For the $74-\mathrm{Hz}$ case $\overline{U^{\prime}}$ increases with pulsation amplitude but $\overline{V^{\prime}}$ shows no similar trend. At $67 \mathrm{~Hz}$, although the range of pulsation amplitude is greater, no trend with pulsation amplitude is seen in $\overline{U^{\prime}}$, and the slight trend in $\overline{U^{\prime}}$ is within the repeatability of the data. Therefore, over the range of pulsation amplitude attainable, it may be concluded that there are no clear trends of either streamwise or transverse mean-turbulence intensity with pulsation amplitude. 
TABLE 2

Variation With Mean Reynolds Number ${ }^{a}$

\begin{tabular}{cccccccc}
\hline & Frequency & $P_{\text {rms }}$ & $U_{\text {osc }-\max }$ & $\bar{U}$ & $U_{\text {osc-max }}$ & $\overline{U^{\prime}}$ & $\overline{V^{\prime}}$ \\
$\overline{\operatorname{Re}}$ & $(\mathrm{Hz})$ & $(\mathrm{kPa})$ & $(\mathrm{m} / \mathrm{s})$ & $(\mathrm{m} / \mathrm{s})$ & $\overline{\bar{U}}$ & $(\mathrm{~m} / \mathrm{s})$ & $(\mathrm{m} / \mathrm{s})$ \\
\hline 3450 & 79 & 6.5 & 75.5 & 14.9 & 5.07 & 5.17 & 1.64 \\
3750 & 83 & 7.4 & 79.3 & 16.3 & 4.87 & 4.82 & 1.73 \\
4400 & 90 & 8.6 & 90.4 & 23.8 & 3.80 & 5.24 & 2.04 \\
\hline
\end{tabular}

${ }^{a}$ All velocities and turbulence intensities are at the tail-pipe centerline, at the base location $(X=540 \mathrm{~mm})$.

\section{Variation With Mean Reynolds Number}

Variation in the mean Reynolds number $(\overline{\operatorname{Re}})$ was obtained by varying the mean mass flow rate of reactants supplied to the combustor, and the results are shown in Table 2. Because the magnitude of the energy release also increased with the mean mass flow rate, the pulsation amplitude increased as well. The same effect was noted by Keller et al. [22] when the timing of the energy release was optimized. The stability limits of the combustor would not allow it to be sufficiently detuned to obtain the same pulsation amplitude for each mass flow rate. Also, although the tailpipe length was invariant at $880 \mathrm{~mm}$, the resonant frequency varied from 79 to $90 \mathrm{~Hz}$ (Table 2), due to temperature and combustion timing effects. In Table 2, $U_{\text {osc }-\max } / \bar{U}, \overline{U^{\prime}}$, and $\overline{V^{\prime}}$ are also presented. Both $U_{\text {osc }-\max }$ and $\bar{U}$ increase with increasing mean Reynolds number; however, their ratio $U_{\text {osc }-\max } / \bar{U}$ decreases monotonically. This is also in agreement with the behavior of the heat transfer enhancement in this flow [2]. Variations in $\bar{U}^{\prime}$ are small and exhibit no clear trend with either $\overline{\mathrm{Re}}$ or the ratio of oscillating and mean velocities. $\overline{V^{\prime}}$, however, shows a small but consistent trend of increasing with increasing $\overline{\mathrm{Re}}$.

\section{Shear Stress}

Time-resolved wall shear stresses were calculated from the product of the viscosity and the time-resolved velocity gradients at the wall. The viscosity was taken to be that of equilibrium combustion products at the wall temperature, and the velocity gradients were obtained from the time- resolved velocity boundary layer profiles, one of which was presented in Fig. 5. In Fig. 13 the time-resolved wall shear stress is plotted against normalized time for various frequencies. All data were taken at the base location of $X=540 \mathrm{~mm}$ except the 101-Hz data, which was taken at $X=$ $340 \mathrm{~mm}$ to avoid entrance effects. As previously discussed, variations with axial position are small. The sign of the shear stress is defined relative to the fluid motion; thus a negative shear stress acts in the negative $X$ direction, against the positive velocity fluid, and a positive shear stress acts in positive $X$ direction, against the negative velocity fluid. With this sign definition, the wall shear stress oscillations in Fig. 13 are in phase with the

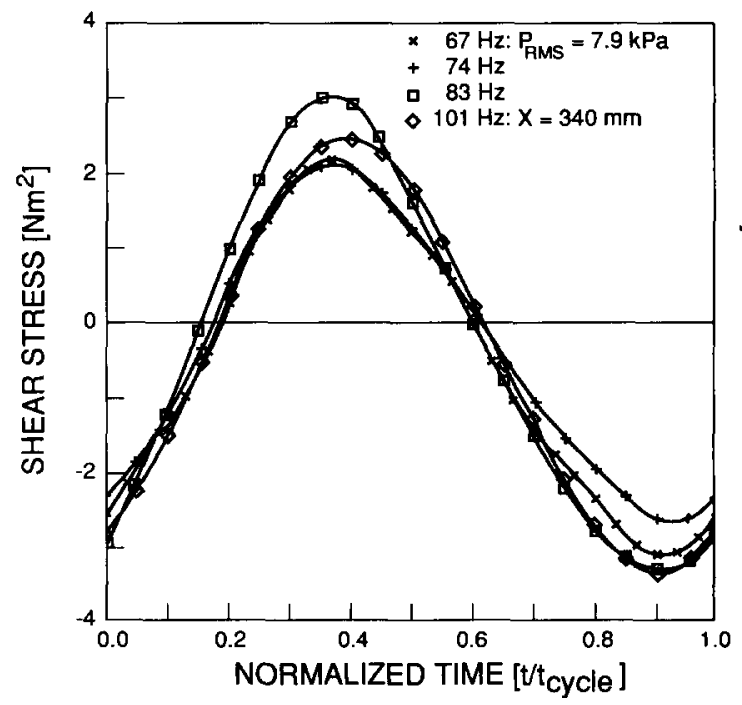

Fig. 13. Wall shear stress as a function of cycle time for various frequencies. $P_{\mathrm{rms}} \approx 7.4 \mathrm{kPa}$ and $X=540 \mathrm{~mm}$, except as noted. 
velocity oscillation near the wall (Fig. 6) and, thus, phase lead the centerline or bulk velocity oscillations by about 0.1 of a cycle. Like the streamwise velocity, the shear stress oscillations are sinusoidal at all frequencies. Little variation with frequency occurs in either the phase or amplitude of these oscillations. (The maximum and minimum values of the shear stresses in Fig. 13 may be slightly overpredicted by a maximum of $15 \%$, because spatial gradient broadening corrections were not applied.) Wall shear stresses were also calculated for various axial locations at the base operating condition. Except for some relatively small entrance and exit effects, little variation in the shear stress with axial position was found, and the behavior seen in Fig. 13 may be considered typical of all axial locations.

Cycle-averaged wall shear stresses from Fig. 13 are presented in Table 1 . This cycle-averaged shear stress is the stress that acts to retard the mean flow of reactants through the tail pipe. For frequencies of 67 and $74 \mathrm{~Hz}$ two pulsation amplitudes were examined, and a trend of increasing cycle-averaged shear stress with pulsation amplitude may be noted. No trend with frequency is noted in this shear stress, which has higher values for 67 and $101 \mathrm{~Hz}$ than at the intermediate frequencies. The average of all the cycle-averaged wall shear stresses in Table 1 is $-0.42 \mathrm{~N} / \mathrm{m}^{2}$, which is slightly greater than the value of -0.38 $\mathrm{N} / \mathrm{m}^{2}$ predicted for steady turbulent flow at the average mean Reynolds number of 3850 . This indicates that the mean-flow momentum transport to the region near the wall in this type of oscillating flow is only slightly greater than that of a steady turbulent flow at the same mean flow Reynolds number.

Also shown in Table 1 are the cycle-averaged absolute-value shear stresses. Since both positive and negative shear stress in this flow retard the fluid motion, this value is a measure of the total work loss, which must be compensated for in order to maintain the oscillations. As with the cycle-averaged values discussed above, cycleaveraged absolute-value shear stresses for the 67and $74-\mathrm{Hz}$ data show a trend of increasing with pulsation amplitude. The cycle-averaged absolute-value shear stresses in Table 1 show a slight trend of being greater at the higher frequencies, but this trend is not sufficiently large to be considered significant. These values average 1.67 $\mathrm{N} / \mathrm{m}^{2}$, which is more than 4 times the expected shear stress of steady turbulent flow at the same mean Reynolds number. Thus, the work input to maintain large reversing velocity oscillations, even for a flow in resonance, is substantially more than for steady turbulent flow. In the pulse combustor this work is input directly from the combustion process, eliminating any additional work input; however, in attempting to use the benefits of large flow oscillations, such as high heat transfer rates $[1,2]$, the cost of supplying the additional work input must be weighed against potential benefits.

Currently, many engineering predictions for oscillating flows are done using quasi-steady assumptions. Under these assumptions, the shear stress at any point in time is taken to be that of steady turbulent flow with a Reynolds number based on the absolute value of the instantaneous velocity. From this definition the cycle-averaged absolute-value shear stress for the base condition and location was calculated to be $3.1 \mathrm{~N} / \mathrm{m}^{2}$, which is 1.6 times greater than the experimentally determined value of $2.0 \mathrm{~N} / \mathrm{m}^{2}$. Comparisons between this quasi-steady result, which is independent of frequency, and the shear stresses for the other frequencies, given in Table 1 , show even greater discrepancies. The poor match of the quasi-steady wall shear stress is not surprising because quasi-steady assumptions are not valid in this flow. The basic assumption that at any point in time the flow behaves as if it were steady flow at the instantaneous Reynolds number, requires that the flow reach fluid-mechanical equilibrium within a time much shorter than the cycle period. This assumption is only valid for flows with very small oscillation amplitudes or very low frequencies. If this assumption were valid; the velocity boundary layer at each point in time would look like a fully-developed turbulent boundary layer. The profiles in Fig. 5 clearly show that this is not the case. Further evidence against the validity of quasi-steady assumptions is the phase leading of the shear stress relative to the bulk velocity oscillation. If the flow is quasi-steady, they must be in 

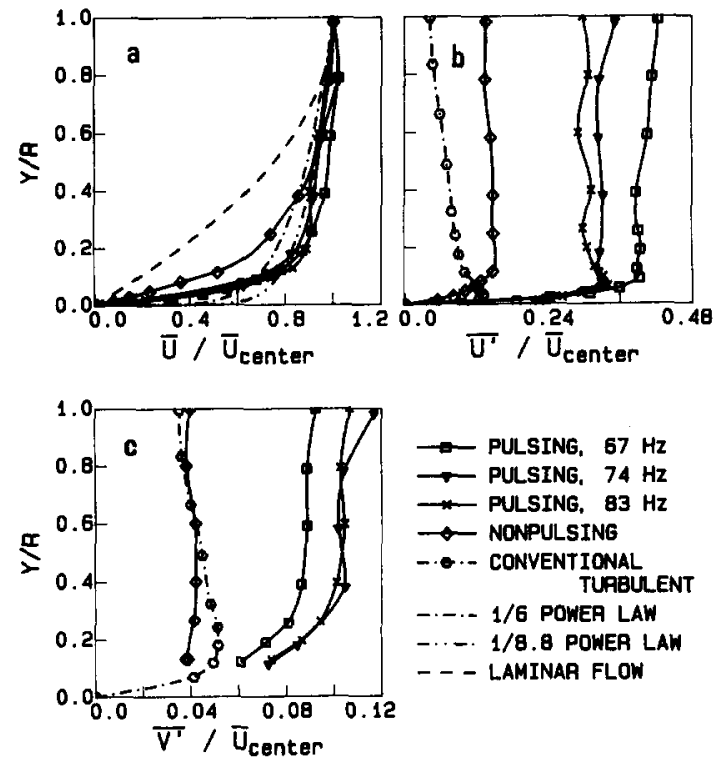

Fig. 14. Comparison of time-averaged pulsing and nonpulsing profiles with those of conventional steady turbulent flow. Figure 14a shows the streamwise velocity, Fig. 14b the streamwise turbulence, and Fig. $14 \mathrm{c}$ the transverse turbulence. The conventional turbulence profiles are adapted from Reichardt [31]. $X=540 \mathrm{~mm}$ and $P_{\mathrm{rms}} \approx 7.4 \mathrm{kPa}$, except for the $67 \mathrm{~Hz}$ case, for which $P_{\mathrm{rms}}=7.9 \mathrm{kPa}$.

phase. The periodically adverse pressure gradients, resulting from the acoustic resonance, also invalidate quasi-steady theory, which assumes flow behavior at all cycle times to be that of steady flow with a favorable pressure gradient. These factors, and the limited accuracy of the quasi-steady shear stresses should be considered in future design calculations.

\section{Comparison of Time-Averaged Profiles with Steady Turbulent Flow}

Time-averaged profiles of $\bar{U}, \overline{U^{\prime}}$, and $\overline{V^{\prime}}$, from the pulsing and nonpulsing flow in the tail pipe are compared with those of conventional steady turbulent flow in Figs. 14a, 14b, and 14c, respectively. All data from the current study were taken at the base location of $X=540 \mathrm{~mm}$, and pulsing data for 67,74 , and $83 \mathrm{~Hz}$ are included. The conventional data are those of Reichardt, as presented by Schlichting [31], which was obtained in a large rectangular duct with a cross section of
$1.0 \mathrm{~m}$ by $0.244 \mathrm{~m}$. These profiles were measured at the center of the duct, in the 0.244-m direction, for a centerline velocity of $1 \mathrm{~m} / \mathrm{s}$ and a Reynolds number of about 15,000 . Reichardt's data were selected for comparison because they were obtained in a rectangular duct, although like most conventional turbulent pipe flow studies, the velocity was lower and the physical dimensions much larger than those of the pulse combustor tail pipe. For ease of comparison of these two different flows, in Fig. 14 all the $Y$ locations have been normalized by $R$, the half height of the rectangular duct (for the pulse combustor tail pipe $R$ is the hydraulic radius or $15 \mathrm{~mm}$ ), and the mean-velocity and turbulence values have been normalized by the mean velocity at the pipe centerline.

In Fig. 14a, mean-velocity profiles for both pulsing and nonpulsing cases are compared with typical profiles for fully developed laminar and turbulent pipe flow. The two turbulent profiles were produced with $1 / 6$ and $1 / 8.8$ power laws, which have been shown to give good fits to experimental data for steady pipe flow with Reynolds numbers of 4000 and $1.1 \times 10^{6}$, respectively [31]. (Reichardt's velocity profile falls between these two curves and is not shown.) It can be seen that the nonpulsing-flow profile falls between the laminar and turbulent solutions, indicating that it is a flow in transition. This result is reasonable for a Reynolds number of 3500 and a smooth pipe, and is in agreement with nonpulsing heat transfer measurements in the combustor [2]. In contrast, the pulsing-flow mean-velocity profiles look similar to the conventional steady turbulent profiles, but with two differences. First, in the core region, these profiles fall to the right of the steady flow profile for a Reynolds number of $1.1 \times 10^{6}$, although the mean Reynolds number is $\mathbf{3 8 5 0}$. This indicates that the mean momentum transport in the core of the pulsing flow is similar to that of steady flow at a much higher mean Reynolds number. Second, the pulsing profiles cross the steady-flow profiles at about $Y / R=$ 0.1 , with the steady flow profiles appearing to have a steeper gradient at the wall. This second difference is believed to be an artifact of the power law approximation for the steady velocity 
profiles, which is known to overpredict the velocity in the viscous dominated region near the wall [31]. The shear stresses, discussed above, also show that the power law approximation is in error near the wall, since the mean shear stress in the pulsing flow slightly greater than the value predicted for steady flow. Finally, caution should be exercised in drawing conclusions from these time-averaged profiles, because they do not provide a complete picture of all the fluid mechanics in this periodically reversing flow.

Mean streamwise turbulence-intensity profiles are shown in Fig. 14b. The most striking difference in these profiles is the large variation in the magnitude of $\overline{U^{\prime}}$ in the core region. The conventional profile has an average normalized intensity in the core of about 0.075 , about half that of the nonpulsing case and 4-6 times less than those of the pulsing flow. These differences would be 15-20 times greater if the turbulence intensities had not been normalized by the time-averaged centerline velocity. Even with this normalization, it is evident that the average streamwise turbulence intensity in the pulsing flow is much greater than that of either the steady flow in this tail pipe, or Reichardt's steady flow, which had a mean Reynolds number nearly 4 times that of the pulsing flow. The turbulence intensity profiles from the current study are also much flatter across the core than the conventional profile, which shows a drop in turbulence intensity by a factor of 2 from $Y / R=0.1$ to the centerline. Uniformity of the turbulence intensity across the core of the pulsing flow again suggests high rates of convective transport in the core. As with the mean velocity profiles, both the pulsing and nonpulsing $\overline{U^{\prime}}$ profiles from this study begin to decrease from their values in the core much farther from the wall than Reichardt's profile. Similar to the mean velocity profiles, this is believed to be an artifact of the large differences in the pipe diameter between the two experiments.

$\overline{V^{\prime}}$ profiles presented in Fig. 14c show similar trends to the $\overline{U^{\prime}}$ profiles, but the differences in magnitude of the core intensities is not as great. In the pulsing flows the core value of $\overline{V^{\prime}}$ is about double that of the nonpulsing flow in the tail pipe or Reichardt's steady flow. As with $\bar{U}$ this dif- ference would be 15-20 times greater if the intensities were not normalized by the centerline velocity. These high transverse turbulence fluctuations may contribute to the high momentum transport in this pulsing flow, which was evidenced in the mean velocity profile shapes and high average shear stress. High $\overline{V^{\prime}}$ intensities may also contribute to the high rates of convective heat transfer, which have been noted in strongly oscillating turbulent flows.

\section{SUMMARY AND CONCLUSIONS}

An experimental study of the velocity field in the tail pipe of a "Helmholtz" type pulse combustor has been conducted. Using LDV, temporally and spatially resolved measurements were made in this strongly oscillating turbulent flow. These flow oscillations, which result from an acoustic resonance, had amplitudes of up to 5 times the mean velocity, and frequencies ranging from 67 to 101 Hz. The streamwise velocity and turbulence boundary layers were measured to within $130 \mu \mathrm{m}$ of the wall, and transverse turbulence measurements were made to within $2 \mathrm{~mm}$.

This study yielded the following results:

1. The bulk velocity oscillations in the tail pipe of a "Helmholtz" type pulse combustor are sinusoidal at the fundamental frequency of the combustor, and are one quarter of a cycle out-of-phase with the combustion-chamber pressure oscillations, as predicted by acoustic theory.

2. At all times during the oscillation cycle, ensemble-averaged velocity profiles are nearly flat across the flow except for a 3- or 4-mm region adjacent to the wall.

3. The velocity oscillations near the wall phaselead the oscillations of the core flow. This phenomenon results in a flow reversal through the boundary layer at cycle times near the zero velocity crossing. The penetration distance of the boundary layer reversals into the flow is approximately the same as the laminar acoustic boundary layer thickness, although the turbulence more rapidly dissipates the oscillations. In this flow the acoustic boundary layer 
thickness is about the same as the time-averaged velocity boundary layer thickness.

4. Oscillations at twice the combustor frequency occur in the streamwise turbulence intensity. In the core, the turbulence-intensity oscillations phase lead the absolute value of the velocity oscillations and are thought to result from flow acceleration effects. Near the wall the oscillations are in phase with the absolute value of the local velocity oscillations, and are thought to result from velocity shear. Transverse turbulence did not vary significantly during the cycle.

5. For the pulsing flow, no significant variation in the velocity field with axial position was found, with the exception of entrance and exit effects. These latter effects, though significant, did not dominate the flow field.

6. Changes in frequency were not found to create any measurable trends in the velocity or transverse-turbulence behavior. Streamwise-turbulence intensities were found to have the general trend of decreasing with increasing frequency.

7. Increases in the mean flow Reynolds number resulted in increases in the pulsation amplitude, but decreases in the ratio of oscillating to mean velocity $\left(U_{\mathrm{osc}-\max } / \bar{U}\right)$. No trends in the streamwise-turbulence behavior were noted, but the transverse-turbulence intensity increased with mean flow Reynolds number.

8. Wall shear stresses oscillate in phase with the velocity near the wall, and therefore phase led the core velocity oscillations by one tenth of a cycle. The amplitude of the shear stress oscillations was found to be 6 times that predicted for steady turbulent flow at the same mean Reynolds number, and the cycle-averaged absolute-value shear stress was more than 4 times the predicted value for steady turbulent flow. Both the cycle-averaged and cycle-averaged absolute-value shear stresses were found to increase with pulsation amplitude, despite the absence of similar trends in the mean transverse-turbulence intensity. No significant trends in shear stress with frequency or axial position were noted.

9. A comparison of time-averaged velocity and turbulence profiles with those of classic steady-turbulent flow was made. The timeaveraged oscillating velocity profiles appeared similar to the steady velocity profile for a Reynolds number much higher than the mean Reynolds number of the oscillating flow. Both streamwise and transverse turbulence profiles were more flat across the core region than those of steady flow, and showed higher intensities than those of the steady flow, indicating a possible mechanism for the increased convective transport that has been noted in strongly oscillating flows.

The authors would like to express their gratitude to Pamela Barr of Sandia for her preparation of Fig. 1. This work was performed at the Combustion Research Facility at Sandia National Laboratories and was supported by the U.S. Department of Energy, Division of Energy Conversion and Utilization Technologies Program. John Dec was supported by the Gas Research Institute. Ichiro Hongo was supported by the Toshiba Corporation.

\section{REFERENCES}

1. Hanby, V. I., ASME J. Eng. Power 91:48-52 (1969).

2. Dec, J. E. and Keller, J. O., Combust. Flame 77:359-374 (1989).

3. Galitseyskiy, B. M., and Ryzhov, Yu. A., Heat Transf. Sov. Res. 9(4): 178-183 (1977).

4. Anon., Proceedings Vol. I: Symposium on Pulse Combination Applications, GRI-82/0009.2, Atlanta, GA, March 1982.

5. Dec, J. E., and Keller, J. O., Combust. Flame 80:358-370 (1990).

6. Putnam, A. A., Belles, F. E., and Kentfield, J. A. C., Prog. Ener. Combust. Sci. 12:43-79 (1986).

7. Zinn, B. T., ASME Winter Annual Meeting, New Orleans, LA, December 1984, Paper 84-WA/NCA-19.

8. Karlsson, S. K. F., J. Fluid Mech. 5:622-636 (1959).

9. Cousteix, J., Desopper, A., and Houdeville, R., in Turbulent Shear Flows I, (Durst, Launder, Schmid, and Whitelaw, Eds.). Springer-Verlag, New York, 1979, pp. 154-171.

10. Cousteix, J., Houdeville, R., and Javelle, J., in Three Dimensional Turbulent Shear Flows I (Carmi, Hamed, Herring, and Peterson, Eds.), ASME, New York, NY, pp. 121-128, 1982.

11. Jayaraman, R., Parikh, P., and Reynolds, W. C., Technical Report No. TF-18, Department of Mechanical Engineering, Stanford University, Stanford, CA, 1982. 
12. Brereton, G. J., and Reynolds, W. C., Technical Report No. TF-29, Department of Mechanical Engineering, Stanford Univ., Stanford, CA, 1987.

13. Mao, Z., and Hanratty, T. J., J. Fluid Mech. 170:545-564 (1986).

14. Keil, R. H., and Baird, M. H. I., Ind. Eng. Chem. Process Des. Dev. 10 (1971).

15. Bogdanoff, D. W., Technical Report No. 483-f, Department of Aerospace and Mechanical Sciences, Princeton University, Princeton, NJ, 1967; also Defense Technical Information Center, Technical Report No. AD-664248.

16. Dec, J. E., and Keller, J. O., Sandia National Laboratories Report, SAND86-8757.

17. Telionis, D. P., Unsteady Viscous Flows, SpringerVerlag, New York, 1981, pp. 222-257.

18. Barr, P. K., Dwyer, H. A., and Bramlette, T. T., Combust. Sci. Technol. 58:315 (1988).

19. Tsujimoto, Y., and Machii, N., presented at Twenty-first Symposium (International) on Combustion, Munich, 1986.

20. Barr, P. K., Keller, J. O., Westbrook, C. K., Bramlette, T. T., and Dec, J. E., Combust. Flame (in press). (Also Sandia National Laboratories Report, SAND89-8221).

21. Keller, J. O., Barr, P. K., Bramlette, T. T., Evens, L. J., and Marchant, R. N., Combust. Sci. Technol. 66:127 (1989).

22. Keller, J. O., Dec, J. E., Westbrook, C. K., and Bramlette, T. T., Combust. Flame 75:33 (1989).

23. Keller, J. O., Westbrook, C. K., Bramlette, T. T., and Dec, J. E., Combust. Flame 79:151-161 (1990).

24. Bramlette, T. T., Sandia National Laboratories Report, SAND87-8622.

25. Keller, J. O., and Saito, K., Combust. Sci. Technol. 53:137 (1987).

26. Kreid, D. K., Appl. Opt. 13:1872-1881 (1974).

27. Morse, A. P., Whitelaw, J. H., and Yianneskis, M., ASME J. Fluids Eng. 101:208-216 (1979).

28. Temkin, S., Elements of Acoustics, Wiley, New York, 1981, pp. 401-417.

42. Rott, N., in Theory of Laminar Flows, (Moore, Ed.), Princeton Univ. Press, 1964 pp. 396-403.

30. White, F. M., Viscous Fluid Flow, McGraw-Hill, New York, 1974, pp. 148-150.

31. Schlichting, H., Boundary Layer Theory, McGrawHill, New York, 6th ed. 1968, pp. 532, 563-565.

Received 27 November 1989; revised 26 June 1990

\section{APPENDIX: TEMPORAL GRADIENT BROADENING CORRECTIONS}

Temporal gradient broadening errors occur as a result of velocity variation across the time bins. Under the assumption of constant seed density in the fluid, the probability of a particle arriving in the probe volume is also proportional to the velocity, which compounds the magnitude of the correction. In the current study the assumption of constant seed density was verified experimentally by examining the number of velocity realizations in the various time bins through the cycle. Good correspondence between the number of realizations and absolute value of the velocity was found. The measured velocity values may be expressed as the integral over the time bin of the product of the true velocity and the probability of a particle arriving in the probe volume, divided by the integral over the time bin of the particle-arrival probability. Assuming constant seed density, the particle-arrival probability is proportional to the true velocity. Expressing the true velocity as a Taylor series expansion about the mean, with second- and higher-order terms neglected, and evaluating the integrals, yields the following equation for the mean velocity in each time bin:

$U_{\text {meas }}=U_{0}+\frac{\tau^{2}}{12 U_{0}}\left(\frac{\partial U_{0}}{\partial t}\right)^{2}$.

A similar development may be followed for the variance, which yields the following result for the turbulence in each time bin:

$$
\begin{aligned}
U_{\text {meas }}^{\prime}= & {\left[U_{0}^{\prime 2}+\frac{\tau^{2}}{12}\left(\frac{\partial U_{0}}{\partial t}\right)^{2}\right.} \\
& \left.-\frac{\tau^{4}}{72 U_{0}^{2}}\left(\frac{\partial U_{0}}{\partial t}\right)^{4}\right]^{1 / 2} .
\end{aligned}
$$

In these equations $U$ and $U^{\prime}$ are, respectively, the ensemble-averaged values of the velocity and turbulence (square root of the variance) in each time bin. $\tau$ is the temporal width of the time bin, and the subscripts meas and 0 correspond to the measured and true values, respectively.

When the velocity oscillations are sufficiently strong to cause flow reversal, two time bins each cycle have both positive and negative velocities in them. These time bins require a special development for the gradient broadening as the sum of two partial time bins, because the probability of a particle arriving in the probe volume is proportional to the absolute value of the velocity, while 
the mean velocity and turbulence depend on the actual velocity value. For these bins the resulting mean velocity and turbulence correction equations are

$$
\begin{aligned}
& U_{\text {meas }}=\frac{\frac{2}{3} U_{0}^{3}+\frac{\tau^{2}}{2} U_{0}\left(\frac{\partial U_{0}}{\partial t}\right)^{2}}{U_{0}^{2}+\frac{\tau^{2}}{4}\left(\frac{\partial U_{0}}{\partial t}\right)^{2}}, \\
& U_{\text {meas }}^{\prime}=\left[U_{0}^{\prime 2}(t)+\frac{\frac{1}{6} U_{0}^{6}+\frac{5 \tau^{2}}{24} U_{0}^{4}(t)\left(\frac{\partial U_{0}}{\partial t}\right)^{2}-\frac{\tau^{4}}{32} U_{0}^{2}(t)\left(\frac{\partial U_{0}}{\partial t}\right)^{4}+\frac{\tau^{6}}{128}\left(\frac{\partial U_{0}}{\partial t}\right)^{6}}{\left(U_{0}^{2}+\frac{\tau^{2}}{4}\left(\frac{\partial U_{0}}{\partial t}\right)^{2}\right)^{2}}\right]
\end{aligned}
$$

Since the mean velocity of the time bin appears in the denominator of the gradient broadening correction, the largest corrections will be made when the velocity is low. As a result, near times of flow reversal the corrections are a large fraction of the measured value, and any errors in the measurement or assumptions required for the correction then become a large fraction of the corrected value.

Figure A1 shows typical corrections for the mean velocity and turbulence data for each time bin through the combustor cycle at the base condition and location. The mean velocity corrections shown in the top plot of Fig. A1 are negligible except near the zero velocity crossing. Even at these times, the correction appears small on this plot; however, note that at times 0.25 and 0.7 the correction cuts the value of the measured velocity nearly in half. This correction of about 3 $\mathrm{m} / \mathrm{s}$ has a significant impact on the shape of the reversing boundary layer profiles. Turbulence corrections in the bottom plot of Fig. A1 are seen to be large over a major portion of the cycle. Again, the corrections are largest in regions of large temporal velocity gradient. Unlike the mean velocity, there is a significant difference in the shape of the corrected and uncorrected turbulence-time plots. After applying these corrections, the velocity oscillations were smooth, and both the velocity and turbulence data were consistent across the tail pipe.
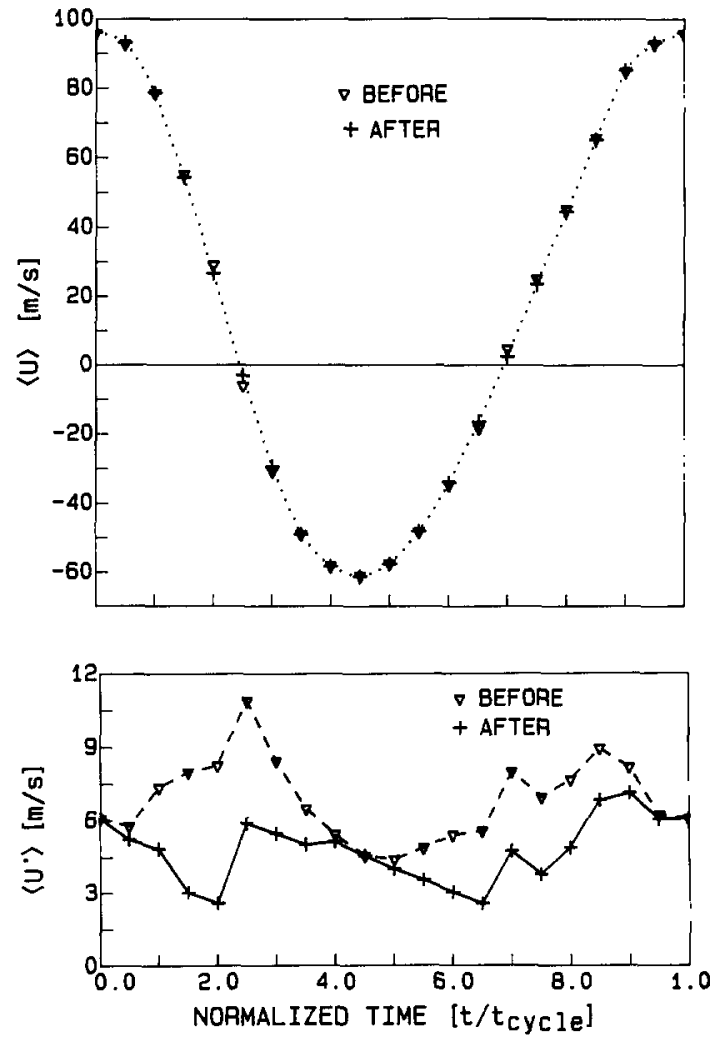

Fig. A1. Temporal-gradient-broadening correction for the streamwise velocity (top) and streamwise turbulence (bottom) data. Base operating condition and base location. 